\title{
AYURLOG
}

National Journal of Research in Ayurved Science

\section{A Literature review on various methods of Ksharsutra preparation}

\author{
Dixit Pankaj ${ }^{1}$, Sonambekar Vinay ${ }^{2}$, Pathak Santosh ${ }^{3}$, Mane Mayuri* ${ }^{4}$
}

1. Associate Professor, Ph. no: 9011045371, Email address: drpankajpdixit@ gmail.com

2. Professor \& HOD, Ph. no: 9822624890, Email address: sonambekar.chinmay@gmail.com

3. Assistant Professor, Ph. no: 9405369727, Email address: drsantoshsp@ gmail.com

4. PG Scholar Shalya Tantra Dept.,

Dept. of Shalya Tantra, A.S.S Ayurved Mahavidyalaya, Nasik, Maharashtra, India 422003

*Corresponding author: Ph. no: 8149767191, Email: dr.mayurimane246@ gmail.com

\begin{abstract}
Fistula-in-ano is a condition which has been recognized as troublesome surgical disease due to its recurrent nature. Almost all the surgeons starting from Acharya Sushruta to the reputed surgeons up to the minute have realized the difficult course of this disorder as far as its surgical as well as medical management is considered. Treatment of fistula-in-ano is always a challenging task for the surgeons and the patients as well. The Ray of hope is provided by Ayurveda in combating this ailment. "Ksharsutra " is an excellent Para surgical procedure for management of Fistula in ano and possible decrease in its reoccurrence. An attempt has been made to review all the references mentioned regarding the preparations of ksharsutras and modifications made in it to improve its utility.
\end{abstract}

Keywords: Ksharsutra, methods, bhagandar, fistula, ayurvedic, seton

\section{Introduction}

"Ksharsutra" is a unique and excellent parasurgical procedure for management of Fistula-in-ano, Hemorrhoids, Warts, Moles, Pilonidal sinus etc. Ksharsutra literally means a thread coated with Kshar. Kshar in Sanskrit means ' one that is capable of destruction". Kshar is based on its property of corroding the skin or the flesh of an affected part of the body.

\section{"क्षरणात दुष्ट त्वक मांसादि चालनात} शातनादीत्यर्थः अन्ये तु क्षरणात दोषाणां चालनात |"

(डल्हण)

Ksharsutra ligature performs excision by virtue of its mechanical pressure and phytochemical cauterization.

This article attempts to compile various methods of the preparation of Ksharsutra from various classical text and 
practitioners. It also includes the new experimentations and formulations which have been published in reputed medical journals.

Ksharsutra is mainly used in the treatment of Fistula-in-ano. Fistula is called as Bhagandara in Ayurveda. As we know, the treatment of fistula aims at excising the complete track by surgery. Fistula i.e. Bhagandara is notoriously known for its recurrence. Sushrut has emphasized this fact as follows ${ }^{1}$.

\section{"घोरा:साधयितुंदुखा:सर्वएवभगंदरा:| \\ तेष्वसाध्य स्तिदोषोत्थः क्षतजश्च \\ भगन्दर:||" \\ (सु.नि.४/१४)}

The treatment of fistula is Fistulectomy or Fistulotomy. Both the surgeries include cutting the whole length of track. For large fistula surgery can cause serious complications like incontinence. Ksharsutra by the virtue of its action prevents this. It is also known to prevent the recurrence. This property and utility is well accepted by the modern science also.

Ksharsutra is a linen thread which is coated with certain Ayurvedic drugs which have corrosive and desloughing actions on an unhealthy granulation tissue which is the root cause of delayed healing of fistula. There are number of such drugs mentioned in the Ayurvedic classical texts.

The first reference of ksharsutra is seen in Sushrut samhita in the chapter of VisarpaNadi-Stanroga Chikitsaadhyay ${ }^{3}$. It says
"कृश दुर्बल भिरूणां नाडी मर्माश्रिता च या| क्षारसूत्रेण तां छिन्द्यान्न तु शस्त्रेण बुद्धिमान् ||"

(सु.चि. ९७/२९)

The treatment of a Nadi Vrana i.e a sinus is actually excision. But if the patient having Nadi vrana is weak, either physically or mentally and if the site of Nadivrana is a Marmasthana, excision is contraindicated. That nadivrana must be treated with Ksharsurtra ${ }^{4}$.

Although the credit of the invention of Ksharsutra is given to Sushruta, there is no clear-cut description of preparation of ksharsutra in Sushrut samhita. The references are found scattered in other samhitas, which have been compiled hereafter.

\section{Material \& methods}

All Information in this article is collected from Ayurvedic texts and journals.

\section{Various methods for Preparation of Ksharsutra}

\section{A. Chakradatta}

The credit of the first distinct description of the manufacturing of ksharsutra is given to Chakrapanidatta. He is known for his legendary commentaries on both Charaka samhita and Sushruta samhita. He has authored one important samhita which is known as Chakradatta ${ }^{8}$. The first reference regarding preparation of ksharsutra is found in this samhita. This is a widely practiced reference by all Ayurvedic practitioners.

The famous reference goes as follows 
"भावितं रजनी चुर्णे: स्नुहि क्षीरै: पुन:

पुनः|बन्धनात सुद्धं सूत्रं छिन्नत्योर्श भगंदरम्|| "

In the fifth Chapter of Arshachikitsa, Acharya Chakradutta has mentioned the use of Ksharsutra in combating Fistula-inano and haemorrhoids ${ }^{8}$.

According to this reference, a thread coated multiple times with Snuhiksheera (the latex of Euphorbia nerifolia) and Rajani churna (Turmeric powder/Curcuma longa) cures Arsha and Bhagandara.

\begin{tabular}{|l|l|l|l|l|l|}
\hline Sr. & Name & Latin name & $\begin{array}{l}\text { Ras - virya- } \\
\text { vipak }\end{array}$ & Guna & Karma \\
\hline 1 & Snuhiksheera & $\begin{array}{l}\text { Latex of } \\
\text { Euphorbia } \\
\text { nerifolia }\end{array}$ & $\begin{array}{l}\text { katu } \\
\text { ushna } \\
\text { katu }\end{array}$ & $\begin{array}{l}\text { Laghu } \\
\text { Tikshna }\end{array}$ & $\begin{array}{l}\text { Lekhan, Vednasthapan } \\
\text { Shothar,Vishagna }\end{array}$ \\
\hline 2 & Rajni churna & $\begin{array}{l}\text { Curcuma } \\
\text { longa }\end{array}$ & $\begin{array}{l}\text { katu, tikta } \\
\text { ushna } \\
\text { katu }\end{array}$ & $\begin{array}{l}\text { Ruksha, } \\
\text { Laghu }\end{array}$ & $\begin{array}{l}\text { Vranashodhana, Kandughna, } \\
\text { Shothhar, Krumighna }\end{array}$ \\
\hline
\end{tabular}

\section{B. $\quad$ Acharya Vangsen}

\section{"स्नुही काण्डगते क्षीरे भल्लातक समन्विते। ज्योतिष्मति त्रिफला दंती कोशातक्यsग्नि}

सैन्धवै: ||

चुर्णैरेतै: समघृतै: बन्धयेत सूत्रकमदढम् |सूत्रं तत्पातयेदर्श: छिन्नमूलं इवद्रुमम ||"

In the chapter of Arsha chikitsa, Acharya Vangsen mentions the utilization of Ksharsutra in the treatment of Arsha ${ }^{12}$. According to him,

\begin{tabular}{|c|c|c|c|c|c|}
\hline No & Name & Latin name & Ras-virya-vipak & Guna & karma \\
\hline 1 & Snuhi & $\begin{array}{l}\text { Euphorbia } \\
\text { nerifolia }\end{array}$ & $\begin{array}{l}\text { katu } \\
\text { ushna } \\
\text { katu }\end{array}$ & $\begin{array}{l}\text { Laghu } \\
\text { Tikshna }\end{array}$ & $\begin{array}{l}\text { Lekhan, Vednasthapan } \\
\text { Shothar,Vishagna }\end{array}$ \\
\hline 2 & $\begin{array}{l}\text { Rajni } \\
\text { churna }\end{array}$ & $\begin{array}{l}\text { Curcuma } \\
\text { longa }\end{array}$ & $\begin{array}{l}\text { Katu tikta } \\
\text { Ushna } \\
\text { katu }\end{array}$ & $\begin{array}{l}\text { Ruksha, } \\
\text { Laghu }\end{array}$ & $\begin{array}{l}\text { Vranashodhana, } \\
\text { Kandughna,Shothhar } \\
\text {,Krumighna }\end{array}$ \\
\hline 3 & $\begin{array}{l}\text { Bhallat } \\
\text { aka } \\
\text { churna }\end{array}$ & $\begin{array}{l}\text { Semecarpus } \\
\text { anacardium }\end{array}$ & $\begin{array}{l}\text { Katu, tikta, kashay } \\
\text { Ushna } \\
\text { madhur }\end{array}$ & $\begin{array}{l}\text { Laghu } \\
\text { tikshna } \\
\text { snigdha }\end{array}$ & $\begin{array}{l}\text { Vatakafahara, } \\
\text { Chedhana, shothar, } \\
\text { krumihar, }\end{array}$ \\
\hline 4 & $\begin{array}{l}\text { Malkan } \\
\text { gani }\end{array}$ & $\begin{array}{l}\text { Celastrus } \\
\text { paniculatus }\end{array}$ & $\begin{array}{l}\text { Katu tikta } \\
\text { Ushna } \\
\text { katu }\end{array}$ & tikshna & vranhara \\
\hline 5 & $\begin{array}{l}\text { Triphal } \\
\text { a: } \\
\text { i)aamal } \\
\text { a }\end{array}$ & $\begin{array}{l}\text { Emblica } \\
\text { officinalis }\end{array}$ & $\begin{array}{l}\text { Madhur, amla, katu, } \\
\text { tikta, kashay. } \\
\text { Sheeta } \\
\text { madhur }\end{array}$ & Guru sheeta & $\begin{array}{l}\text { Daahhar,shothar, } \\
\text { rasayana,vrushya,prame } \\
\text { hagn }\end{array}$ \\
\hline
\end{tabular}




\begin{tabular}{|c|c|c|c|c|c|}
\hline & $\begin{array}{l}\text { ii)behd } \\
a\end{array}$ & $\begin{array}{l}\text { Terminalia } \\
\text { bellirica }\end{array}$ & $\begin{array}{l}\text { Kashay } \\
\text { Ushna } \\
\text { madhur }\end{array}$ & $\begin{array}{l}\text { Ruksha } \\
\text { laghu }\end{array}$ & $\begin{array}{l}\text { Bhedanam, } \\
\text { kruminashanam }\end{array}$ \\
\hline & $\begin{array}{l}\text { iii)harit } \\
\text { aki }\end{array}$ & $\begin{array}{l}\text { Terminal } \\
\text { chebula }\end{array}$ & $\begin{array}{l}\text { Madhur, amla, katu, } \\
\text { tikta, kashay. } \\
\text { ushana } \\
\text { madhur }\end{array}$ & $\begin{array}{l}\text { Laghu } \\
\text { ruksha }\end{array}$ & $\begin{array}{l}\text { Shothar, krumihar, } \\
\text { rasayan }\end{array}$ \\
\hline 6 & Danti & $\begin{array}{l}\text { Baliosperm } \\
\text { um } \\
\text { montanum }\end{array}$ & $\begin{array}{l}\text { Katu } \\
\text { ushna } \\
\text { katu }\end{array}$ & $\begin{array}{l}\text { Guru } \\
\text { tikshna }\end{array}$ & Shothar, vran ropaniya \\
\hline 7 & Chitrak & $\begin{array}{l}\text { Plumbago } \\
\text { zeylanica }\end{array}$ & $\begin{array}{l}\text { Katu } \\
\text { ushna } \\
\text { katu }\end{array}$ & $\begin{array}{l}\text { Laghu } \\
\text { ruksha }\end{array}$ & Shothar, krumihar \\
\hline 8 & $\begin{array}{l}\text { Koshat } \\
\text { aki } \\
(\text { Kadu } \\
\text { dodka) }\end{array}$ & $\begin{array}{l}\text { Luffa } \\
\text { acutangula }\end{array}$ & $\begin{array}{l}\text { Tikta } \\
\text { Ushna } \\
\text { katu }\end{array}$ & $\begin{array}{l}\text { Laghu } \\
\text { ruksha }\end{array}$ & $\begin{array}{l}\text { Shothar, kushtahar, } \\
\text { jwarhar, bhedaniya }\end{array}$ \\
\hline
\end{tabular}

i. Snuhiksheera, Bhallataka churna, Malkangani, Triphala churna, Dantimoola churna, Kadudodkabij churna, Chitrak, Saindhav, etc. are taken in equal quantities

ii. This homogenous mixture is mixed well with Ghrita and applied over linen thread and allowed to dry.

As per the reference there is no guideline for how many coatings are expected. But as it is not mentioned only one coat is expected here.

C. Rasatarangini [Vd.Sadanand Sharma] "सुधा दुगधे वस्त्रपूत हरीद्राचुर्ण संयुते |निषिक्तेन तु तूलेन स्वल्पेन खलु यत्नतः||
प्रलिप्तं सुद्ं सूत्रं च्छायायाम् अथ शोषयेत् | विलीप्य सप्तधाहि एवं शोषयेत्भिषकवर:||

सुत्रम् एतत् समाख्यातं क्षारं सुत्रं तु नामत:|"

According to Rastaringini ${ }^{10}$,

i. Haridra [Turmeric] powder mixed with Snuhiksheera (the latex of Euphorbia nerifolia) is applied on the linen and allowed to dry in a shadow.

ii. This procedure is done for 7 day.

\begin{tabular}{|l|l|l|l|l|l|}
\hline No. & Name & Latin name & $\begin{array}{l}\text { Ras } \\
\text { Virya } \\
\text { vipak }\end{array}$ & Guna & Karma \\
\hline 1 & Snuhiksheera & $\begin{array}{l}\text { Euphorbia } \\
\text { nerifolia }\end{array}$ & $\begin{array}{l}\text { katu } \\
\text { ushna } \\
\text { katu }\end{array}$ & $\begin{array}{l}\text { Laghu } \\
\text { Tikshna }\end{array}$ & $\begin{array}{l}\text { Lekhan, Vednasthapan } \\
\text { Shothar, Vishagna }\end{array}$ \\
\hline 2 & $\begin{array}{l}\text { Rajni churna } \\
\text { haridra }\end{array}$ & $\begin{array}{l}\text { Curcuma } \\
\text { longa }\end{array}$ & $\begin{array}{l}\text { katu, tikta } \\
\text { ushna } \\
\text { katu }\end{array}$ & $\begin{array}{l}\text { Ruksha, } \\
\text { Laghu }\end{array}$ & $\begin{array}{l}\text { Vranashodhana, } \\
\text { Kandughna, } \\
\text { Shothhar, } \\
\text { Krumighna }\end{array}$ \\
\hline
\end{tabular}


D. Ras Kamdhenu ${ }^{7}$

"अर्क स्नुहिपयो: लिप्तं पुन: सूत्रं विशोषयेत्

| त्रिवारान्शोषयित्वा तु क्षारेण एव प्रलेपयेत् II"

i. The thread coated with Snuhiksheera (latex of euphorbia nerifolia) or Arkasheera (latex of Calatropis gigantean), should be dried in shade.

ii. This procedure is repeated three times.

iii. That coated thread after having applied with kshar should be used in rooting out Arsha and Bhagandara.

E. Prof. Dr.P.J. Deshpande (B.H.U.)

Late Prof. Dr.P.J.Deshpande the then Dean,Faculty of Ayurveda, B. H. U, rejuvenated the almost obsolete Ksharsutra - He did an extensive research and standardized the procedure for preparation of Ksharsutra.

i. The surgical linen thread size number 20 is spread throughout the length of Ksharsutra chamber and hung inside Ksharsutra cabinet .

ii. The thread is coated with Snuhiksheera (latex of Euphorbia nerifolia) uniformly all around the linen with the help of clean gauze piece.

iii. After application the Ksharsutra chamber is hung inside the cabinet for drying .Same procedure repeated for 11 days .

iv. On the twelth day,12th coating of Snuhiksheera is done and wet thread is then passed through a heap of finely powdered a ApamargaKshar immediately and hung in the cabinet to dry . This procedure is done continuously for 7 days .

v. The 19th coating is done with Snuhiksheera and fine powder of Haridra churnafor the next 3 days.

vi. As per above said procedure, Ksharsutra is prepared making a total of 21 days coating .

\begin{tabular}{|l|l|}
\hline Day & Daily Coating \\
\hline $\begin{array}{l}\text { Day 1- } \\
11\end{array}$ & Snuhiksheera \\
\hline $\begin{array}{l}\text { Day 12- } \\
18\end{array}$ & Snuhiksheera + ApamargaKshar \\
\hline $\begin{array}{l}\text { Day 19- } \\
21\end{array}$ & Snuhiksheera + Haridra churna \\
\hline
\end{tabular}

\begin{tabular}{|l|l|l|l|l|l|}
\hline No. & Name & Latin name & $\begin{array}{l}\text { Ras } \\
\text { Virya } \\
\text { vipak }\end{array}$ & Guna & karma \\
\hline 1 & Snuhiksheera & $\begin{array}{l}\text { Latex } \\
\text { Euphorbia } \\
\text { nerifolia }\end{array}$ & $\begin{array}{l}\text { katu } \\
\text { ushna } \\
\text { katu }\end{array}$ & $\begin{array}{l}\text { Laghu } \\
\text { Tikshna }\end{array}$ & $\begin{array}{l}\text { Lekhan, Vednasthapan } \\
\text { Shothar, Vishagna }\end{array}$ \\
\hline 2 & $\begin{array}{l}\text { Rajni churna } \\
\text { furcuma longa } \\
\text { churna }\end{array}$ & $\begin{array}{l}\text { katu, tikta } \\
\text { powhna } \\
\text { katu }\end{array}$ & $\begin{array}{l}\text { Ruksha, } \\
\text { Laghu }\end{array}$ & $\begin{array}{l}\text { Vranashodhana, Kandugh } \\
\text { na, } \\
\text { Shothhar, Krumighna }\end{array}$ \\
\hline 3 & $\begin{array}{l}\text { Apamarga } \\
\text { kshar }\end{array}$ & $\begin{array}{l}\text { Achyranthes } \\
\text { aspera }\end{array}$ & $\begin{array}{l}\text { Katu tikta } \\
\text { Ushna } \\
\text { katu }\end{array}$ & $\begin{array}{l}\text { Laghu } \\
\text { ruksha } \\
\text { tikshna }\end{array}$ & $\begin{array}{l}\text { Kaphanashana, } \\
\text { kandughna }\end{array}$ \\
\hline
\end{tabular}


Most of the ksharsutra practitioners use the method standardized by Prof. Dr. P. J. Deshpande. The obsolete ksharsutra has been rejuvenated and now accepted worldwide.

\section{Various Kshara Dravyas-}

There are many herbal drugs which are known for their kshara property. Apamarga kshara is the most widely used kshara. But other drugs also can be used for preparation in ksharsutra.

Bhavprakash and Raj Nighantu give us different groups of ksharas like ksharadwaya, ksharapanchak, ksharashatak, ksharadashak etc.

\begin{tabular}{|l|l|}
\hline क्षारव्दय & सज्जीक्षार,यवक्षार \\
\hline क्षारपंचक & सज्जीक्षार, यवक्षार, तिलक्षार,मुष्ककक्षार, पलाषक्षार \\
\hline क्षारषटक & सज्जीक्षार,यवक्षार, तिलक्षार,मुष्ककक्षार,अपामार्गक्षार, लांगली. \\
\hline क्षाराष्टक & सज्जीक्षार,यवक्षार, तिलक्षार, पलाषक्षार,अपामार्गक्षार, चिंचाक्षार, निवडुंगक्षार,अर्कक्षार \\
\hline
\end{tabular}

\section{Modifications and new research}

Various researchers have modified the way of preparing Ksharsutras. The research has been published in various journals of repute. Here is the compilation.

\begin{tabular}{|l|l|l|l|l|l|}
\hline $\begin{array}{l}\text { Sr. } \\
\text { No. }\end{array}$ & $\begin{array}{l}\text { Reference } \\
\text { taken from }\end{array}$ & Year & Ksharsutra material & Authors & Place \\
\hline 1 & $\begin{array}{l}\text { Journal of } \\
\text { national } \\
\text { remedies }\end{array}$ & 2018 & $\begin{array}{l}\text { Guggul ksharsutra= } \\
\text { Guggul } \\
\text { extract+apamargakshar+ } \\
\text { haridra }\end{array}$ & $\begin{array}{l}\text { Vd. Saxena Varsha } \\
\text { Vd Singh Lakshman }\end{array}$ & $\begin{array}{l}\text { B.H.U. } \\
\text { varanasi }\end{array}$ \\
\hline 2 & $\begin{array}{l}\text { International } \\
\text { journal of } \\
\text { AYUSH }\end{array}$ & 2018 & $\begin{array}{l}\text { Kumari } \\
\text { ksharsutra=kumariswar } \\
\text { as+haridra }\end{array}$ & $\begin{array}{l}\text { Vd } \\
\text { Amarprakash } \\
\text { Vd Bhoir Vedika } \\
\text { Vd PawarAnirudha } \\
\text { Vd Kulkarni Anjana }\end{array}$ & $\begin{array}{l}\text { Navi } \\
\text { mumbai }\end{array}$ \\
\hline 3 & $\begin{array}{l}\text { Internation } \\
\text { journal of } \\
\text { current } \\
\text { research in } \\
\text { life sciences }\end{array}$ & 2018 & $\begin{array}{l}\text { Palash ksharsutra }= \\
\text { Palash + apamarga }+ \\
\text { haridra }\end{array}$ & $\begin{array}{l}\text { Vd Brahmanand Swami } \\
\text { Vd Hiremath Sapana }\end{array}$ & Bidar \\
\hline 4 & $\begin{array}{l}\text { Journal of } \\
\text { Ayurveda \& } \\
\text { integrated } \\
\text { medical }\end{array}$ & $\begin{array}{l}\text { Papaya ksheer based } \\
\text { sphatikaksharsutra }\end{array}$ & $\begin{array}{l}\text { Vd Amulya h. } \\
\text { Vd Shilpa P.N. } \\
\text { Vd Madhunayak R. }\end{array}$ & $\begin{array}{l}\text { karnatak } \\
\text { a }\end{array}$ \\
\hline
\end{tabular}




\begin{tabular}{|c|c|c|c|c|c|}
\hline & $\begin{array}{l}\text { sciences(JAI } \\
\mathrm{MS})\end{array}$ & & & & \\
\hline 5 & $\begin{array}{l}\text { Journal of } \\
\text { Ayurveda \& } \\
\text { integrated } \\
\text { medical } \\
\text { sciences(JAI } \\
\text { MS) }\end{array}$ & 2018 & Tankan ksharsutra & $\begin{array}{l}\text { Vd Ashish Patel } \\
\text { Vd B.S. Savadi }\end{array}$ & $\begin{array}{l}\text { karnatak } \\
\text { a }\end{array}$ \\
\hline 6 & $\begin{array}{ll}\text { Journal of } & \text { Ayurveda \& } \\
\text { integrated } & \\
\text { medical } \\
\text { sciences(JAI } \\
\text { MS) }\end{array}$ & 2018 & Vibhitaki ksharsutra & $\begin{array}{l}\text { Vd Lohitkalal } \\
\text { Vd Akash Kembhavi }\end{array}$ & $\begin{array}{l}\text { Karnatak } \\
\text { a }\end{array}$ \\
\hline 7 & $\begin{array}{l}\text { Journal of } \\
\text { Ayurveda \& } \\
\text { integrated } \\
\text { medical } \\
\text { sciences(JAI } \\
\text { MS) }\end{array}$ & 2017 & $\begin{array}{l}\text { Kadali ksharsutra= } \\
\text { Kadalikshar } \\
\text { arkaksheer }\end{array}$ & $\begin{array}{l}\text { Vd Shilpa Patil } \\
\text { Vd Ashok Naikar } \\
\text { Vd ChandrakanthHalli }\end{array}$ & $\begin{array}{l}\text { karnatak } \\
\text { a }\end{array}$ \\
\hline 8 & $\begin{array}{l}\text { International } \\
\text { journal of } \\
\text { bioassays }\end{array}$ & 2015 & $\begin{array}{l}\text { Arka ksharsutra }= \\
\text { Arka ksheera+ } \\
\text { Apamarga kshar }+ \\
\text { haridra }\end{array}$ & $\begin{array}{l}\text { Vd SatendraTanvar } \\
\text { Vd Shailaja S.V. }\end{array}$ & $\begin{array}{l}\text { Karnatak } \\
\text { a }\end{array}$ \\
\hline 9 & $\begin{array}{l}\text { International } \\
\text { journal of } \\
\text { applied } \\
\text { Ayurved } \\
\text { research }\end{array}$ & 2015 & Snuhi haridra sutra & $\begin{array}{l}\text { Vd Amar Dwivedi } \\
\text { Vd Anaya Pathrikar }\end{array}$ & $\begin{array}{l}\text { Sion } \\
\text { (Mumba) }\end{array}$ \\
\hline 10 & $\begin{array}{l}\text { Innovare } \\
\text { journal of } \\
\text { Ayurvedic } \\
\text { sciences }\end{array}$ & 2013 & Gomutra ksharsutra & $\begin{array}{l}\text { Vd Manojkumar Yadav } \\
\text { Vd Akhilesh Bhargava } \\
\text { Vd H.K.Kushwah }\end{array}$ & Jaipur \\
\hline 11 & JAHM & 2013 & $\begin{array}{l}\text { Madhu (honey) based } \\
\text { ksharsutra }\end{array}$ & $\begin{array}{l}\text { Vd Chakradhar K.V. } \\
\text { Vd Sravanthi S. } \\
\text { Vd Navyakeethana }\end{array}$ & Tirupati \\
\hline
\end{tabular}

Discussion:

Thus there are lot many formulations which are used for the preparation of ksharsutra. Almost all of them are quite effective as per the research papers published. The choice of ksharsutra depends on the individual practitioner.

The utility of ksharsutra has been proven already. It is quite a boon for treatment of difficult fistulae. It does not cause incontinence and also significantly 
reduces the recurrence. Ksharsutra chikitsa has been lauded by modern medicine also thanks to the monumental efforts and dedication put forth by Late Prof. Dr. P. J. Deshpande.

\section{Conclusion:}

Based on this literature review, we would like to conclude by saying that ksharsutras are now being prepared on the basis of various agenda like the availability of certain drugs, diseased condition, patient condition, etc. Thereby a variety of ksharsutras are made in used in clinical practice by making certain modification with the help of authentic method mentioned in the classical texts of Ayurved. ksharsutra shows its own importance and shows marvelous results when applied with proper indications, strict aseptic precautions and extreme carefulness.

\section{References:}

1. Sushrutha Samhita of maharshi susruta, volume II, by Dr.Anant Ram Sharma, foreword by acharya Priyavrat Sharma , Chaukhamba surbharati prakashan, Varanasi, reprint 2015, nidanasthan , bhagandara chikitsaadhyay, verse 14

2. 2.Sushrutha Samhita of maharshi susruta, volume II, by Dr.Anant Ram Sharma, foreword by acharya Priyavat Sharma , Chaukhamba surbharati prakashan, Varanasi,reprint 2015 , chikitsasthan , bhagandara chikitsaadhyay, verse 3

3. Sushrutha Samhita of maharshi susruta, volume II, by Dr.Anant Ram Sharma, foreword by acharya
Priyavrat Sharma, Chaukhamba surbharati prakashan, Varanasi, reprint 2015, chikitsasthan , visarpanadistanrog chikitsaadhyay, verse 29,page no 306-307

4. Sushrutha: Sushrutha Samhita with commentary of Dalhana, edited by Vaidya Jadavaji Trikamji Acharya, Chowakhamba subharti prakashan, Varanasi, reprint 2009, Chikitsa Sthana, 17th Chapter, Verse 29-33, pp-824, pg-468.

5. Charaka Samhita, revised by Charaka \& Dridabala, with Ayurveda Dipika commentary of Chakrapanidatta, Edited by Jadavji Trikamji Acharya; Varanasi: Chaukhambha Prakashan; Reprint 2013; shvaythu chikitsaadhyay, Chikitsasthana 12/96- 97, Pp738, p490.

6. Acharya VruddhaVagbhata, Ashtanga Samgraha; with the Sasilekha commentary by Indu, Prologue by Prof.Jyotir Mitra, dited by Dr. Shivprasad Sharma; Varanasi: Chowkhamba Sanskrit series office; Reprint 2008; Uttarasthana 33/2-44, Pp 965, p797-803

7. Rasakamdhenu with Suvritta by Shri Chudamani Mishra, Hindi Commentry, Edited by Acharya shri Gulraj Mishra; Varanasi: Chowkhamba Orientalia; Chikitsaadhikar 49 bhagandara chikitsa/1-2,Pp 329

8. Chakrapanidatta, Chakradatta; with the Vaiyaprabha Hindi Commentary by Dr. Indradeva Tripathi, Edited by Prof. Ramanath Dwivedy; Varanasi: Chaukhambha Sanskrit Bhawan; Reprint2010; 
Nadivrana chikitsa/12-13, p269272.

9. Dravyaguna Vidnyan part $1 \& 2$, authors Dr.A.P.Deshpande and Dr.R.R.Jawalgekar and Dr.subhash Ranade, profishant publishing house, reprint 2012 , Dravyaguna part 1 , chapter 20. Mishrak gana, kshar, pg no. 297-301

10. Ras-tarinigini by Sharma S. 24th Tarang. 1st ed. Varanasi: Chaukhambha Publishers, shlok 527-530
11. P.J. Deshpande authored the book titled 'Management of Fistula by Kshara Sutra'.

12. vangsen bhashatika Samhita, arshaadhikar, page no.162, shlok no $370-371$

13. https://www.ncbi.nlm.nih.gov/pub med/

14. https://scholar.google.com/

15. https://shodhganga.inflibnet.ac.in/

16. https://www.cochranelibrary.com/

\section{Cite this article:}

A Literature review on various methods of Ksharsutra preparation

Dixit Pankaj, Sonambekar Vinay, Pathak Santosh, Mane Mayuri

Ayurlog: National Journal of Research in Ayurved Science- 2020; (8) (3): 01-09 